\title{
Los cambios a nivel cognitivo como posible mecanismo explicativo del efecto del auto habla sobre el rendimiento
}

\section{Changes on a Cognitive Level as a Mechanism Underlying the Self Talk's Effects on Performance}

\author{
Alexander T. Latinjak*, Miquel Torregrosa** y Jordi Renom*** \\ ${ }^{*}$ Universitat de Girona, Catalunya, España, **Universitat Autònoma de Barcelona, Catalunya, España y ***Universitat de Barcelona, Catalunya, España
}

\begin{abstract}
Resumen: El objetivo principal de este trabajo ha sido aplicar dos tipos de auto habla instruccional y relacionar sus efectos a nivel cognitivo con sus efectos a nivel de rendimiento en una tarea de precisión de tenis. Para ello, 48 tenistas masculinos de ocio $\left(M_{\text {edad }}=36,04\right.$ ańos; D.T. $\left.=6,81\right)$ han sido distribuidos en un grupo de auto habla instruccional tradicional, un grupo de auto feedback, y un grupo control. Todos los participantes han llevado a cabo un ejercicio de derechas cruzadas en línea base y durante la fase de intervención. Hemos medido su acierto, el grado de satisfacción con el rendimiento y su concentración percibida. Los análisis de la covarianza han mostrado mejoras en todas las variables dependientes para los dos grupos de auto habla mientras que no hubo cambios en el grupo control. Además, hemos hallado relaciones significativas entre los cambios en la concentración y en el acierto y la satisfacción. Nuestros resultados indican que ambos tipos de auto habla tienen efectos positivos en la práctica del tenis y que el efecto de la técnica sobre el rendimiento se puede, en parte, explicar a través de sus efectos a nivel cognitivo.

Palabras clave: Tenis, concentración, atención, deportes
\end{abstract}

Abstract: The primary purpose of this study was to apply two types of instructional self talk and to correlate their effects on a cognitive level with their effects on a performance level. Therefore, 48 adult male leisure tennis players $\left(M_{\text {age }}=36.04\right.$ years; S.D. $\left.=6.81\right)$ were divided into three groups: a traditional instructional self talk group, a self feedback group and a control group. All players performed a simple forehand crosscourt exercise in both baseline and intervention phase. Our dependent variables were accuracy, performance satisfaction and perceived concentration. The analyses of covariance showed significant improvements for both self talk groups regarding all three dependent variables, whilst no changes were registered for the control group. Moreover, we found a significant correlation between the changes in concentration and the changes in accuracy and satisfaction. Overall, our results evidence positive effects of both instructional self talk types on the practice of Tennis and they support the idea that the effects of self talk on performance could, partially, be explained by its effects on concentration.

Key words: Tennis, attention, concentration, sports

\section{Introduccción}

El auto habla se ha definido como "lo que las personas se decían a sí mismas, ya sea en voz alta o como una voz dentro de sus cabezas" (Theodorakis, Weinberg, Natsis, Douma, y Kazakas, 2000) y se ha identificado como una de las técnicas cognitivas más usadas en psicología del deporte (Chroni, Perkos, y Theodorakis, 2007).

En referencia a los efectos del auto habla, Hatzigeorgiadis, Zourbanos, Galanis y Theodorakis (2011) han publicado un meta-análisis que evidencia sus capacidades para facilitar el aprendizaje y aumentar el rendimiento. No obstante, también han destacado que el tamaño del efecto de la técnica depende del grado en el cual se adecúa el tipo de auto habla al tipo de tarea. En este sentido, el auto habla instruccional sería más eficaz en tareas que requieren precisión y el auto habla motivacional lo sería en tareas que requieren esfuerzo o resistencia.

Dirección para correspondencia: Alexander T. Latinjak, Escola Universitaria de la Salut i de l'Esport (EUSES), c/ Francesc Macià 65, 17190 Salt-Girona, Espańa. E-mail: alexander.latinjak@cadscrits.udg.edu

Esta investigación se ha llevado a cabo en parte gracias a una subvención del Ministerio de Ciencia e Innovación (DEP2010-15561) y de la Fundación Privada EUSES.
También en el Tenis encontramos estudios que han estudiado los efectos de ambos tipos de auto habla. Sus resultados evidencian efectos beneficiosos sobre el aprendizaje de la técnica (e.g., Ziegler, 1987), la ejecución de la técnica (e.g., Cutton y Landin, 2007), la precisión de los golpes (e.g., Latinjak, Torregrosa, y Renom, 2010a), la concentración (e.g., Landin y Hebert, 1999), la auto-eficacia (e.g., Hatzigeorgiadis, Zourbanos, Goltsios, y Theoroakis, 2008) y la ansiedad (e.g., Hatzigeorgiadis, Zourbanos, Mpoumpaki, y Theoroakis, 2009).

Al mismo tiempo que unos estudios han consolidado la evidencia a favor de los efectos beneficiosos del auto habla, otros estudios se han fijado en identificar los mecanismos a través de los cuales la técnica ayuda al rendimiento (Hardy, 2006). En esta línea, Hatzigeorgiadis et al. han obtenido resultados que indican que los cambios a nivel de rendimiento se pueden explicar a través de cambios a nivel de la autoeficacia (Hatzigeorgiadis, Zourbanos, Goltsios et. al, 2008), confianza (Hatzigeorgiadis, Zourbanos, Mpoumpaki et al., 2009) y ansiedad (Hatzigeorgiadis, Zourbanos, y Theoroakis, 2007).

El objetivo principal de este trabajo ha sido aplicar el auto 
habla y relacionar sus efectos a nivel cognitivo con sus efectos a nivel de rendimiento en una tarea de precisión de tenis. Siguiendo las recomendaciones de Hatzigeorgiadis et al., (2011) hemos optado a emplear el tipo instruccional para la tarea de precisión. Además, considerando las evidencias de Cutton y Landin (2007) y Latinjak, Torregrosa y Renom (2011a) acerca de las ventajas de combinaciones entre auto habla instruccional y feedback, hemos usado un auto feedback a parte de la versión tradicional del auto habla instruccional.

En base a las evidencias previas referentes al efecto del auto habla sobre el rendimiento y la concentración (e.g., Latinjak, Torregrosa, y Renom, 2009), esperamos una mejora en el rendimiento y la concentración para ambos grupos de auto habla, mientras que un grupo control no presente cambios. Además, esperamos que los cambios a nivel de concentración en los grupos de auto habla se relacionen positivamente con los cambios a nivel de rendimiento.

\section{Método}

\section{Participantes}

Cuarenta y ocho tenistas masculinos de ocio han participado en nuestro estudio $\left(M_{\text {edad }}=36,04\right.$ ańos; D.T. $\left.=6,81\right)$. Nuestros participantes provenían de un club de tenis municipal del área de Barcelona donde practicaron el tenis una media de 2,71 horas por semana $(S D=, 90)$. Ninguno de ellos ha tomado clases de tenis con un entrenador los dos ańos previos al experimento. Informamos a todos nuestros participantes que la participación fue voluntaria y que pudieran abandonar el estudio en cualquier momento, y ellos firmaron el consentimiento antes de iniciar la sesión experimental.

\section{Procedimiento}

La sesión experimental constaba de cuatro partes: familiarización con la tarea experimental, la recogida de datos en la línea base, introducción y familiarización con el auto habla y la recogida de datos en la fase con intervención. En referencia a la tarea experimental, los participantes habían de devolver 20 pelotas provenientes de una maquina lanza-pelotas con la derecha desde su línea de fondo al área ubicado entre la línea de saque y la línea de fondo del campo contrario. Además, todas las derechas habían de ser cruzadas, por lo que el área objetivo tuvo un tamańo de 22,62 $\mathrm{m}^{2}$.

En referencia a la manipulación experimental, dividimos nuestra muestra en tres grupos: un grupo de auto habla instruccional tradicional (AHI), un grupo de auto feedback (AFB), y un grupo control (CON). El grupo AHI siguió un procedimiento habitual (ver por ejemplo, Latinjak et al., 2010a) mientras que el grupo CON fue instruido en seguir jugando como siempre. La diferencia entre el grupo AHI y el grupo AFB fue la siguiente: El primero creaba a partir de ideas centrales (e.g., flexiona las rodillas) palabras clave (e.g., Rodilla) que se verbalizaban en un momento clave de la ejecución del golpe (e.g., al impactar con la pelota); mientras que el segundo mantuvo en mente la idea central a la hora de ejecutar el golpe para darse, una vez terminado el golpe, auto afirmación (e.g., bien) si ha podido aplicar la idea central (e.g., ha flexionado las rodillas) o auto negación (e.g., mal) si no lo ha conseguido (e.g., siente que no ha flexionado las rodillas).

\section{Materiales y medidas}

El experimento se llevó a cabo en pistas regulares de tenis y los participantes usaron sus propias raquetas. Un maquina lanza-pelotas (Lobster Elite Freedom) fue ubicada en el centro de la línea de fondo en frente del jugador, y un cono fue colocada en el centro del área objetivo.

La medida objetiva del rendimiento ha sido la variable acierto. En cada repetición los jugadores han puntuado de 0 a 20 , en función del número de golpes que habían acertado a su área objetivo. Después de cada repetición de la tarea experimental, los jugadores han completado los datos objetivos sobre el rendimiento a través de una pregunta sobre la satisfacción con el rendimiento. Han valorado su satisfacción en una escala de 0 a 10 que iba desde nada satisfecho a totalmente satisfecho. Además, para medir la concentración, los jugadores se han auto evaluado en otra escala de once puntos que iba desde 0 = nada concentrado en absoluto a $10=$ completamente concentrado. Finalmente, los jugadores del ambos grupos de intervención valoraban también en qué medida habían aplicado el auto habla durante el ejercicio en una escala de 1 (nunca) a 6 (siempre).

\section{Análisis de datos}

En primer lugar, hemos controlado el grado de cumplimiento con la intervención a través de la interpretación de la variable control. En segundo lugar, hemos usado un análisis de la varianza simple para analizar las diferencias entre los tres grupos en la línea base, para garantizar la validez de los análisis posteriores. En tercer lugar, hemos usado tres análisis de la covarianza para analizar el efecto de la manipulación experimental sobre las variables acierto, satisfacción y concentración. En todos los análisis de la varianza hemos calculado también el tamańo de los efectos significativos $\left(\eta^{2}\right)$ : hemos tomado los valores de $\eta^{2}=, 010 ; \eta^{2}=, 059$; y $\eta^{2}=, 138$ como correspondientes a efectos pequeños, medianos, y grandes, respectivamente (Kirk, 1996). Para completar el análisis hemos usado comparaciones múltiples entre los tres grupos para todos los efectos significativos. Para terminar, hemos correlacionado los cambios a nivel de rendimiento, objetivo y subjetivo, y a nivel de concentración. Para ello hemos seguido 
el procedimiento de Hatzigeorgiadis et al. (2007) que han sustraído las puntuaciones de la línea base a las de la fase con intervención.

\section{Resultados}

\section{Análisis preliminares}

Los datos descriptivos de la variable control indican que los participantes han aplicado la técnica más que casi siempre en las dos fases con auto habla (valores promedios para la aplicación de la técnica; $M=5,59, D T=, 61$ en el AHI y $M=5,63$, $D T=, 47$ en el grupo AFB). En cuanto a las diferencias entre los tres grupos en la línea base, los análisis no han revelado efectos significativos del factor grupo sobre las variables acierto, satisfacción y concentración (todas las $p>, 05$ ).

\section{El efecto de la manipulación experimental}

En referencia a la variable acierto, hemos encontrado un efecto grande del factor Grupo, $F(2,44)=6,18, p=, 004, \eta^{2}=$ ,219. Este resultado indica que los jugadores de los grupos de intervención han acertado más que los jugadores del grupo CON (ambas $p<, 05$; Valores promedio 13,56 [D.T. $=2,44]$, $12,41[D . T .=2,38]$, y $11,00[D . T .=3,18]$ para los grupos AHI, AFB y CON respectivamente). En referencia a la variable satisfacción, hemos encontrado un segundo efecto grande del factor Grupo, $F(2,44)=16,32, p<, 001, \eta^{2}=, 426$. Este resultado indica que los jugadores de los grupos de intervención se han valorado más satisfechos con su rendimiento que los jugadores del grupo CON (ambas $p<, 001$; Valores promedio 7,25 [D.T. $=1,20], 7,91[D . T .=0,95]$, y 5,50 [D.T. $=$ $1,46]$ para los grupos AHI, AFB y CON respectivamente). En referencia a la variable concentración, hemos encontrado un tercer efecto grande del factor Grupo, $F(2,44)=21,48, p$ $<, 001, \eta^{2}=, 494$. Este resultado indica que los jugadores de los grupos de intervención se han valorado más concentrados que los jugadores del grupo CON (ambas $p<, 001$; Valores promedio 7,69 [D.T. = 0,93], 8,22 [D.T. = 0,71], y 6,28 [D.T. $=1,41]$ para los grupos AHI, AFB y CON respectivamente). Además, los jugadores del grupo AFB también se han valorado más concentrados que los jugadores del grupo AHI ( $p$ $=, 011)$.

\section{La relación entre los cambios a nivel de rendimiento y concentración}

Hemos hallado relaciones positivas y significativas entre los cambios a nivel de rendimiento y concentración: acierto con concentración, $r=0,560, p<, 001$; y satisfacción con concentración, $r=, 661, p<, 001$. Este resultado indica en ambos casos que los aumentos en la concentración en los grupos de intervención se relacionan con las mejoras en el rendimiento.

\section{Discusión}

Nuestros resultados evidencian que ambos grupos de auto habla han sido superiores al grupo control a nivel de rendimiento y concentración, y que los cambios a nivel cognitivo se relacionaron positivamente con los cambios a nivel de rendimiento. En este sentido, dichos resultados son consistentes con las evidencias previas referentes a los efectos positivos del auto habla sobre el rendimiento (ver, Hatzigeorgiadis et al., 2011) y diversas variables cognitivas (e.g., Landin y Hebert, 1999; Latinjak, Torregrosa, y Renom, 2009, 2010b, 2011b; Hatzigeorgiadis et al, 2007).

En este estudio hemos empleado una combinación entre auto habla y feedback, que se basa en las evidencias de Cutton y Landin (2007) sobre los efectos positivos de la combinación entre ambos y que fue presentado en el trabajo de Latinjak, et al. (2011a). Nuestros resultados evidenciaron un funcionamiento similar entre esta nueva versión del auto habla y la tradicional, aunque los jugadores del grupo de auto feedback también valoran más alto su concentración comparado con los del grupo de auto habla tradicional. Es posible que el auto feedback pudiera tener ventajas sobre la versión tradicional porque se reduce la carga de tarea secundaria que supone verbalizar mientras que se ejecute el movimiento.

Además, hemos hallado una relación entre los efectos del auto habla sobre la concentración y sobre el rendimiento. Este resultado indica que el efecto de la técnica sobre el rendimiento se puede, en parte, explicar a través de sus efectos a nivel cognitivo. Otros estudios previos ya han proporcionado evidencias acerca de los mecanismos subyacentes a los efectos sobre el rendimiento (e.g., Hatzigeorgiadis et al., 2007), aunque aún faltan estudios experimentales que confirmen estos resultados (Hatzigeorgiadis, Zourbanos, Mpoumpaki et al., 2009).

Hay algunas limitaciones que deben tenerse en cuenta al interpretar los resultados de este trabajo: no hemos controlado el uso del auto habla durante las repeticiones de la tarea experimental sin intervención; no hemos controlado el uso de otro tipo de auto habla que puede ocurrir en paralelo durante la intervención; y con la variable acierto hemos considerado sólo una pequeña parte del rendimiento.

En resumen, este trabajo ha evidenciado los efectos del auto habla sobre el rendimiento y apoya los cambios cognitivos como mecanismo explicativo de los mismos. Consecuentemente, recomendamos el uso y estudio del auto habla por parte de deportistas, entrenadores y psicólogos de deporte. 


\section{Referencias}

Chroni, S., Perkos, S., y Theodorakis, Y. (2007). Functions and preferences of motivational and instructional ST for adolescent basketball players. Athletic Insight: The Online Journal of Sport Psychology, 9(1), 1-13.

Cutton, D., y Landin, D. (2007). The effects of self-talk and augmented feedback on learning the tennis forehand. Journal of Applied Sport Psychology, 19(3), 288-303.

Hardy, J. (2006). Speaking Clearly: A critical review of self talk literature. Psychology of Sport \& Exercise, 7, 81-97.

Hatzigeorgiadis, A., Zourbanos, N., Galanis, E., y Theodorakis, Y. (2011). Self-talk and sport performance: A meta-analysis. Perspectives on Psychological Science, 6(4), 348-356.

Hatzigeorgiadis, A., Zourbanos, N., Goltsios, C., y Theodorakis, Y. (2008). Investigating the functions of self talk: the effects of motivational self talk on self-efficacy and performance in young tennis players. The Sport Psychologist, 22, 458-471.

Hatzigeorgiadis, A., Zourbanos, N., Mpoumpaki, S., \& Theodorakis, Y. (2009). Mechanisms underlying the self-talk-performance relationship: The effects of motivational self-talk on self-confidence and anxiety. Psychology of Sport and Exercise, 10, 186-192.

Hatzigeorgiadis, A., Zourbanos, N., y Theodorakis, Y. (2007). The moderating effects of self talk content on self talk functions. Journal of Applied Sport Psychology, 19(2), 240-251.

Kirk, R. E. (1996). Practical significance: A concept whose time has come. Educational and Psychological Measurement, 56, 746-759.
Landin, D., \& Hebert, E. P. (1999). The influence of self talk on the performance of skilled female tennis players. Journal of Applied Sport Psychology, 11(2), 263-282.

Latinjak, A. T., Torregrosa, M., \& Renom, J. (2009). Aplicando el autohabla al tenis: su impacto sobre el foco atencional y el rendimiento. Cuadernos de Psicología del Deporte, 9(2), 19-29.

Latinjak, A. T., Torregrosa, M., \& Renom, J. (2010a). El papel de la exigencia de la tarea en la aplicación del auto-habla y su efecto en tenistas de ocio. Revista de Psicología del Deporte, 19(2), 187-201.

Latinjak, A. T., Torregrosa, M., \& Renom, J. (2010b). Studying the effects of self talk on thought contents with male adult tennis players. Perceptual and Motor Skills, 111(1), 249-260.

Latinjak, A. T., Torregrosa, M., \& Renom, J. (2011a). Combining self talk and performance feedback: their effectiveness with adult tennis players. The Sport Psychologist, 25(1), 18-31.

Latinjak, A. T., Torregrosa, M., \& Renom, J. (2011b). Comparing the effects of different self-instructions on thought content and tennis performance. Athletic Insight Journal, 3(1), 17-28.

Theodorakis, Y., Weinberg, R., Natsis, P., Douma, E., y Kazakas, P. (2000) The effects of motivational versus instructional self talk on improving motor performance. The Sport Psychologist, 14, 253-272.

Ziegler, S. (1987). Effects of stimulus cueing on the acquisition of groundstrokes by beginning tennis players. Journal of Applied Behavior Analysis, 20(4), 405-411. 